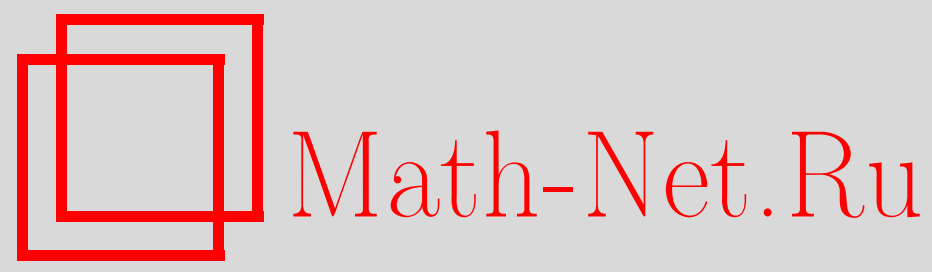

А. В. Аминова, Д. А. Калинин, Алгебры Ли $H$-проективных движений келеровых многообразий постоянной голоморфной секционной кривизны, Матем. заметки, 1999, том 65, выпуск 6, 803-809

DOI: https://doi.org/10.4213/mzm1115

Использование Общероссийского математического портала Math-Net.Ru подразумевает, что вы прочитали и согласны с пользовательским соглашением http://www . mathnet.ru/rus/agreement

Параметры загрузки:

IP : 107.22 .136 .117

26 апреля 2023 г., 14:38:11

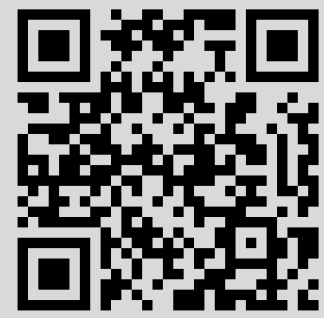




\title{
АЛГЕБРЫ ЛИ $H$-ПРОЕКТИВНЫХ ДВИЖЕНИЙ КЕЛЕРОВЫХ МНОГООБРАЗИЙ ПОСТОЯННОЙ ГОЛОМОРФНОЙ СЕКЦИОННОЙ КРИВИЗНЫ
}

\section{А. В. Аминова, Д. А. Калинин}

\begin{abstract}
В статье рассматриваются алгебры Ли инфинитезимальных $H$-проективных преобразований $2 n$-мерньх келеровых многообразий постоянной голоморфной секционной кривизны. Доказывается, что эти алгебры изоморфны овеществлению комплексной алгебры Ли $\mathrm{sl}(n, \mathbb{C})$, и дается их локальная реализация в виде алгебр векторных полей на многообразии.
\end{abstract}

Библиограффия: 9 названий.

Келеровы многообразия играют существенную роль в различных областях математики и математической физики: теории динамических систем, алгебраической геометрии, геометрии многообразий Эйнштейна, квантовой механике, квантовой теории поля, теории относительности, теории суперструн и нелинейных сигма-моделей. Важным разделом келеровой геометрии является теория $H$-проективных отображений [1]. $H$-проективные отображения представляют собой частньй случай почти геодезических отображений, которые в свою очередь обобшают геодезические отображения псевдоримановых многообразий [2]-[4]. Особьй интерес представляет изучение $H$-проективных отображений келеровых многообразий постоянной голоморфной секционной кривизны, так как такие и только такие келеровы многообразия допускают $H$-проективные отображения на плоское пространство [4], [5]. Кроме того, келеровы многообразия постоянной голоморфной секционной кривизны представляют интерес с точки зрения их приложений в теоретической физике [6], [7].

В данной статье рассматриваются алгебры Ли инфинитезимальных $H$-проективных (голоморфно-проективных) преобразований $2 n$-мерных келеровых многообразий постоянной голоморфной секционной кривизны. Доказьвается, что такие алгебры изоморфны овеществлению комплексной алгебры Ли $\mathrm{sl}(n, \mathbb{C})$. Дается локальная реализация алгебр инфинитезимальных $H$-проективных преобразований в виде алгебр векторных полей на многообразии.

Напомним [8], что әрмитовым многообразием назьвается дифференцируемое многообразие $M$, на котором заданы псевдориманова метрика $g$ и комплексная структура $J$, т.е. дифференцируемое поле операторов $J_{p}: T_{p} M \rightarrow T_{p} M,\left(J_{p}\right)^{2}=-\mathrm{id}_{T_{p}} M$, $p \in M$, для которого тензор Нейенхейса

$$
N(X, Y)=2([J X, J Y]-[X, Y]-J[X, J Y]-J[J X, Y])
$$

Работа выполнена при частичной финансовой поддержке Российского фонда фундаментальных исследований, грант № 96-01-01031. 
обрашается в 0 для любых векторных полей $X, Y$ на $M$. Антисимметричная билинейная форма, определенная равенством $\omega(X, Y)=g(J X, Y)$ для любых векторных полей $X, Y$ на $M$, назьвается фундаментальной 2-формой эрмитова многообразия $M$. Келеровым многообразием назьвается эрмитово многообразие $M$, фундаментальная 2 -форма $\omega$ которого замкнута: $d \omega=0$. В комплексных координатах $z^{\alpha}, \overline{z^{\alpha}}$ на $M$ компоненты келеровой метрики $g$ имеют вид

$$
g_{\alpha \bar{\beta}}=\partial_{\alpha \bar{\beta}} \Phi
$$

где $\Phi$ - вещественнозначная функция, называемая келеровым потенииалом метрики $g$.

Пусть $M$ - келерово многообразие, $\pi_{p}$ - неизотропная плоскость в касательном пространстве $T_{p} M$ к многообразию $M$ в точке $p$, т.е. плоскость такая, что $g(X, X) \neq 0$ для любого ненулевого вектора $X \in \pi_{p}$. Предположим, что плоскость $\pi_{p}$ инвариантна относительно действия оператора $J$, т.е. для любого вектора $X \in \pi_{p}$ вектор $J X$ также принадлежит $\pi_{p}$. Величина

$$
K\left(\pi_{p}\right)=R(X, J X, X, J X), \quad|g(X, X)|=1,
$$

где $R$ - тензор кривизны келерова многообразия $M$, назьвается голоморфной секиионной кривизной многообразия $M$ в направлении $\pi_{p}$. Пользуясь свойствами тензора кривизны, можно показать [8], что голоморфная секционная кривизна $K\left(\pi_{p}\right)$ не зависит от выбора единичного вектора $X \in \pi_{p}$. Если величина $K\left(\pi_{p}\right)$ постоянна для всех $\pi_{p} \subset T_{p} M$ и не зависит от выбора точки $p \in M$, то $M$ назьвается многообразием постоянной голоморфной секиионной кривизны.

Гладкая кривая $\gamma:[0,1] \rightarrow M: t \mapsto x_{t}$ на келеровом многообразии $M$ назьвается аналитически планарной кривой, или $H$-планарной кривой, если она удовлетворяет уравнению

$$
\nabla_{\chi} \chi=a(t) \chi+b(t) J \chi, \quad \chi \equiv \frac{d x}{d t}
$$

где $a(t), b(t)$ - гладкие функции параметра $t$ и $\nabla$ - риманова связность метрики $g$. Пусть $(M, g, J)$ и $\left(M^{\prime}, g^{\prime}, J^{\prime}\right)$ - два келеровых многообразия. Диффеоморфизм $f: M \rightarrow M^{\prime}$ назьвается голоморфно-проективным отображением, или $H$-проективным отображением, если для любой $H$-планарной кривой $\gamma$ в $M$ кривая $f$ о $\gamma$ является $H$-планарной кривой в $M^{\prime}$.

Пусть $M, M^{\prime}$ - два келеровых многообразия и $f: M \rightarrow M^{\prime}$ есть $H$-проективное отображение. Системы локальных координат (карт) $(\Omega, x)$ и $\left(f(\Omega), x^{\prime}\right)$ в $M$ и $M^{\prime}$ назьваются соответственными, если координаты точек $p \in M$ и $f(p) \in M^{\prime}$ совпадают в этих картах. Напомним, что по определению отображение $f: M \rightarrow M^{\prime}$ сохраняет комплексную структуру $J$, если выполняется равенство $f_{*} \circ J=J^{\prime} \circ f$, где $f_{*}$ означает дифференциал отображения $f$. Поскольку любое $H$-проективное отображение сохраняет комплексную структуру [4], в $M$ и $M^{\prime}$ можно ввести соответственные комплексные координаты $(\Omega, z),\left(f(\Omega), z^{\prime}\right)$.

Если келерово многообразие $(M, g)$ с римановой связностью $\nabla$ допускает $H$-проективное отображение на келерово многообразие $\left(M^{\prime}, g^{\prime}\right)$ с римановой связностью $\nabla^{\prime}$, то говорят, что связности $\nabla, \nabla^{\prime} H$-проективно-эквивалентны. Необходимоеи достаточное условие $H$-проективной эквивалентности связностей $\nabla$ и $\nabla^{\prime}$ выражается в локальных координатах равенством

$$
\Gamma_{j k}^{\prime i}-\Gamma_{j k}^{i}=\rho_{j} \delta_{k}^{i}+\rho_{k} \delta_{j}^{i}-\rho_{s} J_{j}^{s} J_{k}^{i}-\rho_{s} J_{k}^{s} J_{j}^{i},
$$


где $\Gamma_{j k}^{i}, \Gamma_{j k}^{\prime i}-$ коэффициенты связностей $\nabla$ и $\nabla^{\prime}, \rho=\rho_{i} d x^{i}-$ некоторая замкнутая $(d \rho=0)$ 1-форма на $M$.

Теорема 1 [1], [5]. Келерово многообразие $M_{2 n}$ является $Н$-проективно-плоским, т.е. допускает Н-проективное отображение на (плоское) келерово пространство $\mathbb{C}^{n}$, тогда и только тогда, когда оно является многообразием постоянной голоморфной секционной кривизны.

Пусть $(M, g, J)$ - келерово многообразие. Вещественноеголоморфноевекторноеполе $X$ (т.е. векторное поле, обладающее свойством $L_{X} J=0$ ) называется инфинитезимальным $H$-проективным преобразованием, или $H$-проективным движением в $M$, если в локальных координатах на $M$ вьполняется условие

$$
L_{X} \Gamma_{j k}^{i}=\varphi_{j} \delta_{k}^{i}+\varphi_{k} \delta_{j}^{i}-\varphi_{s} J_{j}^{s} J_{k}^{i}-\varphi_{s} J_{k}^{s} J_{j}^{i}
$$

где $L_{X}$ - производная Ли, а $\varphi=\varphi_{i} d x^{i}$ есть замкнутая вещественная 1-формана $M$. Так как согласно лемме Пуанкаре [9] любая замкнутая дифференциальная форма локально точна, в окрестности каждой точки $p \in M$ существует функция $\psi$ такая, что $\varphi=d \psi$; следовательно, локально $\varphi_{i}=\partial_{i} \psi$. Если $\varphi \equiv 0$, то $H$-проективное движение назьвается аффинным движсением в $M$. В противном случае, т.е. при $\varphi \neq 0, X$ назьвается собственно $H$-проективным движениемв $M$.

Теорема 2. Для любых двух $H$-проективных движений $X_{(1)} u X_{(2)}$ в келеровом многообразии $(M, g, J)$ их скобка Ли $X=\left[X_{(1)}, X_{(2)}\right]=L_{X_{(1)}} X_{(2)}$ также есть $H$-проективное движение в $M$.

ДокАЗАтЕльство. Так как векторные поля $X_{(1)}, X_{(2)}$ являются $H$-проективньми движениями, в локальных координатах выполняются равенства

$$
\begin{gathered}
L_{X_{(A)}} \Gamma_{j k}^{i}=\varphi_{(A) j} \delta_{k}^{i}+\varphi_{(A) k} \delta_{j}^{i}-\varphi_{(A) s} J_{j}^{s} J_{k}^{i}-\varphi_{(A) s} J_{k}^{s} J_{j}^{i} \\
L_{X_{(A)}} J=0, \quad A=1,2, \quad i, j, k=1, \ldots, 2 n .
\end{gathered}
$$

Отсюда, пользуясь известной формулой

$$
L_{\left[X_{(1)}, X_{(2)}\right]}=L_{X_{(1)}} L_{X_{(2)}}-L_{X_{(2)}} L_{X_{(1)}}
$$

находим

$$
L_{X} \Gamma_{j k}^{i}=\widetilde{\varphi}_{j} \delta_{k}^{i}+\widetilde{\varphi}_{k} \delta_{j}^{i}-\widetilde{\varphi}_{s} J_{j}^{s} J_{k}^{i}-\widetilde{\varphi}_{s} J_{k}^{s} J_{j}^{i},
$$

где $\widetilde{\varphi}=L_{X_{(1)}} \varphi_{(2)}-L_{X_{(2)}} \varphi_{(1)}$. Следовательно, векторноеполе $X=\left[X_{(1)}, X_{(2)}\right]$ является $H$-проективными движением.

Из доказанного следует, что множество всех H-проективных движений келерова многообразия $M$ образует алгебру Ли $\mathrm{hp}(M)$, назьваемую $H$-проективной алгеброй Ли в $M$.

Теорема 3. Пусть $\nabla u \nabla^{\prime}-H$-проективно-эквивалентные связности в $М$. Если $X$ есть инфинитезимальное $H$-проективное преобразование связности $\nabla$, то таким оно является и для связности $\nabla^{\prime}$. Поэтому Н-проективные алгебры Н-проективно-эквивалентных связностей изоморфны. 
ДокАЗАТЕЛЬСТво. Пусть векторное поле $X$ является инфинитезимальным $H$-проективньм преобразованием келерова многообразия $(M, g, J)$. Тогда выполняется условие (2). Если связности $\nabla$ и $\nabla^{\prime} H$-проективно-эквивалентны, то справедливо равенство (1). Взяв производную Ли вдоль векторного поля $X$ от обеих частей этого равенства и используя (2), получим

$$
L_{X} \Gamma_{j k}^{\prime i}=\varphi_{j}^{\prime} \delta_{k}^{i}+\varphi_{k}^{\prime} \delta_{j}^{i}-\varphi_{s}^{\prime} J_{j}^{s} J_{k}^{i}-\varphi_{s}^{\prime} J_{k}^{s} J_{j}^{i}
$$

где $\varphi^{\prime}=\varphi-L_{X} \rho$. Следовательно, $X$ является инфинитезимальньм $H$-проективным преобразованием связности $\nabla^{\prime}$, что и требовалось доказать.

В комплексных координатах $z^{\alpha}, \overline{z^{\alpha}}, \alpha=1, \ldots, n$, определенных в некоторой области $\Omega \subset M$, уравнение (2) можно записать в виде

$$
\begin{gathered}
L_{X} \Gamma_{\beta \lambda}^{\alpha}=2 \delta_{\beta}^{\alpha} \psi_{, \lambda}+2 \delta_{\lambda}^{\alpha} \psi_{, \beta}, \quad L_{X} \Gamma_{\bar{\beta} \lambda}^{\bar{\alpha}}=2 \delta_{\beta}^{\alpha} \psi_{, \bar{\lambda}}+2 \delta_{\lambda}^{\alpha} \psi_{, \bar{\beta}}, \\
L_{X} \Gamma_{\beta \lambda}^{\bar{\alpha}}=L_{X} \Gamma_{\bar{\beta} \lambda}^{\bar{\alpha}}=L_{X} \Gamma_{\overline{\beta \lambda}}^{\alpha}=L_{X} \Gamma_{\bar{\beta} \lambda}^{\alpha}=0,
\end{gathered}
$$

где (вещественнозначная) функция $\psi$ связана с 1-формой $\varphi$ условием $\varphi=d \psi$.

Пусть $(M, g, J)$ - келерово многообразие постоянной голоморфной секционной кривизны $k$. Согласно теореме 1 многообразие $M$ (локально) допускает $H$-проективное отображение на плоское пространство. Поэтому риманова связность $\nabla$ келеровой метрики $g H$-проективно-эквивалентна плоской связности и, следовательно, $H$-проективная алгебра Ли $\mathrm{hp}(M)$ многообразия $M$ изоморфна $H$-проективной алгебре $\mathrm{hp}\left(\mathbb{C}^{n}\right)$ плоского келерова пространства $\mathbb{C}^{n}$, причем в соответственных координатах локальные выражения для базисных векторных полей алгебр Ли $\mathrm{hp}(M)$ и $\mathrm{hp}\left(\mathbb{C}^{n}\right)$ совпадают.

Уравнения $H$-планарной кривой пространства $\mathbb{C}^{n}$ в аффинной системе координат, в которой все символы Кристоффеля плоской метрики равны 0, имеют вид

$$
\frac{d^{2} z^{\alpha}}{d t^{2}}=c(t) \frac{d z^{\alpha}}{d t}, \quad \frac{d^{2} \overline{z^{\alpha}}}{d t^{2}}=\overline{c(t)} \frac{d \overline{z^{\alpha}}}{d t}
$$

где $c(t)$ есть комплекснозначная функция вещественного параметра $t$. Интегрируя эти уравнения, находим

$$
z^{\alpha}=C_{1}^{\alpha} f(t)+C_{2}^{\alpha}, \quad \overline{z^{\alpha}}=\overline{C_{1}^{\alpha}} \overline{f(t)}+\overline{C_{2}^{\alpha}},
$$

где

$$
f(t)=\int d t \exp \left(\int c(t) d t\right),
$$

a $C_{1}^{\alpha}$ и $C_{2}^{\alpha}$ - постоянные интегрирования. Отсюда следует, что $H$-планарные кривые принадлежат подмногообразию $P \subset \mathbb{C}^{n}$, определенному уравнениями

$$
z^{\alpha}=C_{1}^{\alpha} u+\mathrm{C}_{2}^{\alpha}, \quad u \in \mathbb{C}
$$

Пусть $X$ - вещественное $H$-проективное движение в $\mathbb{C}^{n}$ :

$$
X=\xi^{\alpha}(z) \partial_{\alpha}+\overline{\xi^{\alpha}(z)} \partial_{\bar{\alpha}}
$$


где $\xi^{\alpha}(z), \alpha=1, \ldots, n,-$ голоморфные функции. Подставляя это равенство в (3), находим

$$
\begin{gathered}
L_{X} \Gamma_{\beta \lambda}^{\alpha}=\partial_{\alpha} \partial_{\lambda} \xi^{\alpha}=\delta_{\beta}^{\alpha} \psi_{, \lambda}+\delta_{\lambda}^{\alpha} \psi_{, \beta}, \quad L_{X} \Gamma_{\beta \lambda}^{\bar{\alpha}}=\partial_{\bar{\alpha}} \partial_{\bar{\lambda}} \xi^{\bar{\alpha}}=\delta_{\beta}^{\alpha} \psi_{, \bar{\lambda}}+\delta_{\lambda}^{\alpha} \psi_{, \bar{\beta}} \\
L_{X} \Gamma_{\beta \lambda}^{\bar{\alpha}}=L_{X} \Gamma_{\bar{\beta} \lambda}^{\alpha}=L_{X} \Gamma_{\beta \bar{\lambda}}^{\alpha}=L_{X} \Gamma_{\beta \bar{\lambda}}^{\bar{\alpha}}=0 .
\end{gathered}
$$

Условия интегрируемости этих уравнений в комплексных координатах $z^{\alpha}, \overline{z^{\alpha}}$ можно записать в виде равенств

$$
\begin{aligned}
& \left(L_{X} \Gamma_{\beta[\lambda}^{\alpha}\right)_{, \mu]}=2 \psi_{\beta, \mu} \delta_{\lambda}^{\alpha}-2 \psi_{\beta, \lambda} \delta_{\mu}^{\alpha} \equiv L_{X} R^{\alpha} \beta \lambda \mu \\
& \left(L_{X} \Gamma_{\beta[\lambda}^{\alpha}\right)_{, \bar{\mu}]}=2 \psi_{\beta, \bar{\mu}} \delta_{\lambda}^{\alpha}+2 \psi_{\beta, \lambda} \delta_{\bar{\mu}}^{\alpha} \equiv L_{X} R^{\alpha} \beta \lambda \bar{\mu}
\end{aligned}
$$

(квадратные скобки означают альтернирование). Остальные условия интегрируемости уравнений (4) могут быть получены из приведенных вьше с помошью комплексного сопряжения и свойств симметрии тензора кривизны.

Ввиду того что $R_{j k l}^{i}=0$ для $\mathbb{C}^{n}$, из (5) следуют соотношения

$$
\partial_{\alpha} \psi, \beta=0, \quad \partial_{\bar{\alpha}} \psi, \beta=0, \quad \alpha, \beta=1, \ldots, n,
$$

из которых вытекает, что $\psi, \alpha$ и $\psi, \bar{\alpha}$ постоянны для любого $\alpha=1, \ldots, n$, т.е.

$$
\psi=c_{\alpha} z^{\alpha}+\overline{c_{\alpha}} \overline{z^{\alpha}}
$$

Подставив этот результат в (4), получим

$$
\xi^{\alpha}=c_{\sigma} z^{\sigma} z^{\alpha}+a_{\sigma}^{\alpha} z^{\sigma}+b^{\alpha}, \quad \alpha=1, \ldots, n,
$$

где $a_{\sigma}^{\alpha}, b^{\alpha}$ и $c^{\alpha}-$ произвольные комплексные постоянные. Эта формула определяет общий вид $H$-проективного движения $X=\xi^{\alpha}(z) \partial_{\alpha}+\overline{\xi^{\alpha}(z)} \partial_{\bar{\alpha}}$ в плоском пространстве $\mathbb{C}^{n}$ и (в соответственных координатах) в произвольном $2 n$-мерном келеровом многообразии $M$ постоянной голоморфной секционной кривизны. Чтобы получить базисные векторные поля алгебры Ли $\mathrm{hp}\left(\mathbb{C}^{n}\right) \cong \mathrm{hp}(M)$, положим в (6) последовательно

1) $c_{\lambda}=\psi_{\lambda}^{(\mu)}=\eta \delta_{\lambda}^{\mu}, a_{\lambda}^{\alpha}=0, b^{\alpha}=0$;

2) $c_{\lambda}=0, a_{\lambda}^{\alpha}=a_{\lambda(\nu)}^{\alpha(\mu)}=\eta \delta_{\lambda}^{\alpha} \delta_{\nu}^{\mu}, b^{\alpha}=0$;

3) $c_{\lambda}=0, a_{\lambda}^{\alpha}=0, b_{(\mu)}^{\alpha}=\eta \delta_{\mu}^{\alpha}$,

где $\alpha, \lambda, \mu, \nu=1, \ldots, n$, а постоянная $\eta$ равна единице или мнимой единице. Выделенным случаям соответствуют следующие три группы базисных векторных полей:

$$
\begin{gathered}
X^{\mu}=z^{\mu} z^{\sigma} \partial_{\sigma}+\overline{z^{\mu} z^{\sigma}} \partial_{\bar{\sigma}}, \quad Y^{\mu} \equiv J X^{\mu}=i\left(z^{\mu} z^{\sigma} \partial_{\sigma}-\overline{z^{\mu} z^{\sigma}} \partial_{\bar{\sigma}}\right) \\
U_{\nu}^{\mu}=z^{\mu} \partial_{\nu}+\overline{z^{\mu}} \partial_{\bar{\nu}}, \quad V_{\nu}^{\mu}=i\left(z^{\mu} \partial_{\nu}-\overline{z^{\mu}} \partial_{\bar{\nu}}\right) \\
Z_{\mu}=\partial_{\mu}+\partial_{\bar{\mu}}, \quad W_{\mu}=i\left(\partial_{\mu}-\partial_{\bar{\mu}}\right), \quad \mu, \nu=1, \ldots, n .
\end{gathered}
$$

Векторные поля из первой групшы $(X, Y)$ являются собственно $H$-проективньми движениями пространства $\mathbb{C}^{n}$, а векторные поля из второй $(U, V)$ и третьей $(Z, W)$ групш образуют алгебру Ли а $J\left(\mathbb{C}^{n}\right)$ аффинных движений в $\mathbb{C}^{n}$, сохраняющих комплексную структуру. Из (7) следует, что $\operatorname{dim}_{\mathbb{R}} \mathrm{hp}\left(\mathbb{C}^{n}\right)=2 n^{2}+4 n$.

Доказана 
ТеоремА 4. H-проективная алгебра Ли $\mathrm{hp}(M)$ келерова многообразия $M$ постоянной голоморфной секционной кривизны есть $\left(2 n^{2}+4 n\right)$-мерная вещественная алгебра Ли. Генераторами әтой алгебры Ли являются векторные поля $X^{\mu}, Y^{\mu}$, $U_{\nu}^{\mu}, V_{\nu}^{\mu}, Z^{\mu}, W^{\mu}, \mu, \nu=1, \ldots, n$, определенные в подходящей комплексной карте $\left(\Omega, z^{\alpha}, z^{\bar{\alpha}}\right)$ формулами (7).

Известно [8], что в келеровом многообразии $(M, g, J)$ постоянной ненулевой голоморфной секционной кривизны $k \neq 0$ можно ввести локальную комплексную систему координат $z^{\alpha}, \overline{z^{\alpha}}, \alpha=1, \ldots, n$, в которой (ненулевые) компоненты $g_{\alpha \bar{\beta}}$ метрики $g$ и ее келеров потенциал $\Phi$ определяются равенствами

$$
\begin{gathered}
g_{\alpha \bar{\beta}}=\frac{2}{k} \frac{\left(1+\sum_{\sigma} \varepsilon_{\sigma} z^{\sigma} \overline{z^{\sigma}}\right) \varepsilon_{\alpha} \delta_{\beta}^{\alpha}-\varepsilon_{\alpha} \varepsilon_{\beta} \overline{z^{\alpha}} z^{\beta}}{\left(1+\sum_{\sigma} \varepsilon_{\sigma} z^{\sigma} \overline{z^{\sigma}}\right)^{2}}, \quad \varepsilon_{\sigma}= \pm 1 \\
\Phi=\frac{2}{k} \ln \left(1+\sum_{\sigma} \varepsilon_{\sigma} z^{\sigma} \overline{z^{\sigma}}\right)
\end{gathered}
$$

Отличные от 0 символы Кристоффеля метрики $g$ имеют вид

$$
\Gamma_{\beta \gamma}^{\alpha}=\overline{\Gamma_{\bar{\beta}}^{\bar{\alpha}}}=-\mathscr{A}^{-1}\left(z^{\bar{\alpha}} \delta_{\gamma}^{\beta}+z^{\bar{\beta}} \delta_{\gamma}^{\alpha}\right)
$$

где $\mathscr{A}=1+\sum_{\sigma} \varepsilon_{\sigma} z^{\sigma} z^{\bar{\sigma}}$. Вычислив производную Ли от символов Кристоффеля вдоль векторных полей $X^{\mu}, Y^{\mu}, U_{\nu}^{\mu}, V_{\nu}^{\mu}, Z^{\mu}, W^{\mu}, \mu, \nu=1, \ldots, n$, легко убедиться в том, что векторные поля $Z^{\mu}, W^{\mu}, \mu=1, \ldots, n$, являются собственно $H$-проективньми движениями в $M$, а векторные поля $X^{\mu}, Y^{\mu}, U_{\nu}^{\mu}, V_{\nu}^{\mu}, \mu, \nu=1, \ldots, n$, натягивают алгебру Ли а $J(M)$ голоморфных аффинных движений в $M$, т.е. аффинных движений, сохраняющих комплексную структуру.

Запишем базисные векторные поля $X^{\mu}, Y^{\mu}, U_{\nu}^{\mu}, V_{\nu}^{\mu}, Z^{\mu}, W^{\mu}, \mu, \nu=1, \ldots, n$, в вещественных координатах $x^{i}, i=1, \ldots, 2 n$, определенных в области $\Omega \subset M$ равенствами

$$
x^{\alpha}=\frac{1}{\sqrt{2}}\left(z^{\alpha}+\overline{z^{\alpha}}\right), \quad x^{n+\alpha}=\frac{1}{i \sqrt{2}}\left(z^{\alpha}-\overline{z^{\alpha}}\right) .
$$

Выразив $\partial_{\alpha}$ и $\partial_{\bar{\alpha}}$ через $\partial / \partial x^{i}$, из (7) получаем

$$
\begin{gathered}
X^{\mu}=\sqrt{2} \sum_{\sigma}\left(\left(x^{\mu} x^{\sigma}-x^{n+\mu} x^{n+\sigma}\right) \frac{\partial}{\partial x^{\sigma}}+\left(x^{\mu} x^{n+\sigma}+x^{n+\mu} x^{\sigma}\right) \frac{\partial}{\partial x^{n+\sigma}}\right) \\
Y^{\mu}=\sqrt{2} \sum_{\sigma}\left(-\left(x^{\mu} x^{n+\sigma}+x^{n+\mu} x^{\sigma}\right) \frac{\partial}{\partial x^{\sigma}}+\left(x^{\mu} x^{\sigma}+x^{n+\mu} x^{n+\sigma}\right) \frac{\partial}{\partial x^{n+\sigma}}\right) \\
U_{\nu}^{\mu}=x^{\mu} \frac{\partial}{\partial x^{\nu}}+x^{n+\mu} \frac{\partial}{\partial x^{n+\nu}}, \quad V_{\nu}^{\mu}=x^{\mu} \frac{\partial}{\partial x^{n+\nu}}-x^{n+\mu} \frac{\partial}{\partial x^{\nu}} \\
Z_{\mu}=\sqrt{2} \frac{\partial}{\partial x^{\mu}}, \quad W_{\mu}=-\sqrt{2} \frac{\partial}{\partial x^{n+\mu}} .
\end{gathered}
$$

Рассмотрим алгебру Ли $\mathrm{p}\left(\mathbb{C}^{n}\right)$ проективных движений комплексного линейного пространства $\mathbb{C}^{n}$ (т.е. преобразований в $\mathbb{C}^{n}$, которые переводят комплексныепрямые в $\mathbb{C}^{n}$ в 
комплексные прямые). Аналогично вещественному случаю генераторы проективной алгебры соответствуют сдвигам, аффинным преобразованиям и собственно проективным преобразованиям в $\mathbb{C}^{n}$. Этими генераторами являются голоморфные векторные поля

$$
T_{\nu}=\partial_{\nu}, \quad R_{\nu}^{\mu}=z^{\mu} \partial_{\nu}, \quad P^{\mu}=z^{\mu} z^{\sigma} \partial_{\sigma}, \quad \mu, \nu=1, \ldots, n
$$

Как известно [5], алгебра $\mathrm{p}\left(\mathbb{C}^{n}\right)$ изоморфна алгебре Ли $\operatorname{sl}(n+1, \mathbb{C})$. Сравнивая $(7)$ и $(8)$, мы приходим к вьводу, что алгебра $h \mathrm{p}(M)$ является овеществлением алгебры Ли $\mathrm{p}\left(\mathbb{C}^{n}\right) \cong \mathrm{sl}(n+1, \mathbb{C})$.

ТЕорема 5. Н-проективная алгебра $\mathrm{hp}(M)$ келерова многообразия постоянной голоморфной секиионной кривизны изоморфна овеществлению $\mathrm{sl}_{\mathbb{R}}(n+1, \mathbb{C})$ алгебрь $\pi u \operatorname{sl}(n+1, \mathbb{C})$.

\section{СПИСОК ЦИТИРОВАННОЙ ЛИТЕРАТУРЫ}

[1] Otsuki T., Tashiro Y. On curves in Kählerian spaces // Math. J. Okayama Univ. 1954. V. 4. P. $57-78$.

[2] Аминова А. В. Псевдоримановы многообразия с общими геодезическими // УМН. 1993. T. 48. C. $107-159$.

[3] Синюков Н. С. Геодезические отображения римановых пространств. М.: Наука, 1979.

[4] Синюков Н. С., Курбатова И. Н., Микеш Й. Голоморфно-проективные отображения келеровых пространств. Одесса: Изд. Одесского ун-та, 1985.

[5] Ishihara S. Holomorphically projective changes and their groups in an almost complex manifolds // Töhoku Math. J. 1957. V. 9. P. 273-297.

[6] Aminova A. V., Kalinin D. A. Quantization of Kähler manifolds admitting $H$-projective mappings // Tensor. 1995. V. 56. P. 1-11.

[7] Калинин Д. А. Келеровы магнитные поля на пространствах постоянной голоморфной секционной кривизны // Изв. вузов. Физика. 1996. № 1. С. 13-19.

[8] Кобаяси Ш., Номидзу К. Основы дифференциальной геометрии. Т. 1, 2. М.: Наука, 1981.

[9] Постников М. М.Лекции по геометрии. Семестр III. Гладкие многообразия. М.: Наука, 1987. 